\title{
A Unified Equation of Interactions
}

\author{
Hasan Arslan \\ Physics Department, Bingöl University, Bingöl, Turkey \\ E-mail: hasanarslan46@yahoo.com \\ Received July 13, 2011; revised August 14, 2011; accepted August 25, 2011
}

\begin{abstract}
The aim of this study is to combine four fundamental forces in a single equation. Dirac equation is written by putting the Yukawa potential as a representation of the strong and gravitational forces. The ordinary terms seen in the Dirac Equation are treated as the representations of the electromagnetic forces. The Lagrangian of the weak local interaction of the charged particles is converted to the energy representation according to the virial theorem and is put in the equation. Thus four fundamental forces are combined in a unique equation.
\end{abstract}

Keywords: GUT Scale, Fundamental Forces, Dirac Equation, Virial Theorem

\section{Introduction}

After the foundations of the Maxwell's equations, physicists are trying to combine the four fundamental forces in a unique equation. Albert Einstein studied on this subject for a long time but he didn't obtain a unified equation [1]. In [2], the classical solutions of a unified theory is written. A unified description of long-ranged interactions is done in [3]. Some other studies related to a unified Equation are [4-6].

The models at Grand Unified Theories (GUT) implies that at high energy level of the universe the four fundamental forces (weak, electromagnetic, strong, gravitational forces) have the same validity. Therefore, the four fundamental forces must be written in a single representtation to describe the true universe.

The weak interactions are the results of the charged particles and $\mathrm{W}$, and $\mathrm{Z}$ bosons are the force carriers of the weak interactions. The weak interactions are short ranged interactions. Photons are the force carriers in the electromagnetic interactions, the electromagnetic interactions are the long ranged interactions. The strong interactions are confined in the nucleus, it holds nucleons together, three colored gluons are the force carriers in the strong interactions. The gravitational forces are the results of the mass effects of the particles and they are the long ranged interactions.

The non-relativistic Schrödinger equation is written in relativistic form by Dirac in 1928 so named as Dirac equation. It contains electromagnetic interactions of particles.

Yukawa potential is used to describe the strong inter- actions at nuclei distances and the gravitational interactions at large distances.

In the Standard Model, the weak interactions are described by the Lagrangian of the interacting charged particles.

In this study, the Yukawa potential is placed in the Dirac equation and it is solved for the characteristic wave function of the strong and the gravitational interactions. The strong and the gravitational interactions are thought to be combined in a space of higly mass concentrated. Later, the Lagrangian of the weak interactions is written in terms of energy by using the virial theorem. This term is put in the equation and four fundamental forces are written in a single equation.

\section{Materials and Methods}

Previous works and known equations of interactions in physics are used in this study to combine the four fundamental forces. Mathematical calculations are done. The literature is searched. Theoretical assumptions are done. As a result, the combination of four fundamental forces in a single equation is written.

\section{Calculations}

The two strong candidates for a unified theory of interactions are the Klein-Gordon equation and the Dirac equation. The Dirac equation includes the electromagnetic interactions. The Dirac equation is derived in $[7,8]$ as

$$
i \hbar \frac{\partial \psi}{\partial t}=\left[c \vec{\alpha} \cdot\left(\vec{p}-\frac{e}{c} \vec{A}\right)+e \Phi+\beta m c^{2}\right] \psi
$$


where

$$
\alpha_{i}=\left(\begin{array}{cc}
0 & \sigma_{i} \\
\sigma_{i} & 0
\end{array}\right) \text { and } \beta=\left(\begin{array}{cc}
1 & 0 \\
0 & -1
\end{array}\right)
$$

and $\sigma_{i}$ are the $2 \times 2$ Pauli matrices. The first and second terms on the right of the Equation (1) includes kinematical interactions. Since the electromagnetic forces are the result of the kinematical interactions, it is the representation of the electromagnetic forces in the equation. The last term is for the rest mass of the particle.

In [9], the strong and the gravitational interaction potential is given in the form

$$
-\frac{Q^{2}}{r} e^{-r / r_{q}}
$$

here $r_{q}$ is the radius related to the particle under consideration. The total energy is given in the article [9] by

$$
E \approx m c^{2} \frac{R}{r} e^{r / R}
$$

where $R$ is the Schwarzschild radius of the particle. $Q$ is given by the equation

$$
Q^{2}=\gamma m c^{2} r_{q}
$$

here $\gamma=1 / \sqrt{1-v^{2} / c^{2}}$.

The Yukawa potential given by Equation (3) can be directly placed in the Dirac equation;

$$
i \hbar \frac{\partial \psi}{\partial t}=\left[\left[c \boldsymbol{\alpha} \cdot\left(\vec{p}-\frac{e}{c} \boldsymbol{A}\right)\right]+e \Phi+\beta m c^{2}-\frac{Q^{2}}{r} e^{-r / r_{q}}\right] \psi
$$

When the kinematical and charge effects are much higher than the strong and the gravitational effects, then the Yukawa potential must have small effects on the motion of the particles. In this situation, the Yukawa potential term can be omitted and the original Dirac equation explains the motion of the particles.

When $r_{q}$ is too large, the effective energy comes from the gravitational forces. The other terms in Equation (6) are negligible. In this case, the Dirac equation reduces to the form;

$$
i \hbar \frac{\partial \psi}{\partial t}=-\frac{\gamma m c^{2} r_{q}}{r} e^{-r / r_{q}} \psi
$$

Now, the distances can be taken as $r_{q} \sim r$. Then, the Equation (7) reduces to the equation below

$$
i \hbar \frac{\partial \psi}{\partial t}=-\gamma m c^{2} e^{-1} \psi
$$

Integrating Equation (8) we obtain the wave function $\psi$ as

$$
\psi \sim e^{i \gamma m c^{2} t / e \hbar}
$$

This is the wave character of the gravitational forces.
When $r_{q}$ is too small, the effective force on the motion is the strong interaction. Again in this case, $r_{q} \sim r$. The Dirac equation for this condition is the same as Equation (7). Therefore, the wave function given by Equation (9) describes the wave structures of both the gravitational and the strong interactions.

In a highly charged and highly mass concentrated space, the gravitational interaction valid for the large distances and the strong interaction valid for the small distances have the same equivalence. The interaction of the particles for a so much charged, mass concentrated, and higly energitic space can be described by a single equation as

$$
i \hbar \frac{\partial \psi}{\partial t}=\left[\left[c \boldsymbol{\alpha} \cdot\left(\vec{p}-\frac{e}{c} \boldsymbol{A}\right)\right]+e \Phi+\beta m c^{2}-2 \frac{Q^{2}}{r} e^{-r / r_{q}}\right] \psi
$$

where the factor 2 describes the combination of the strong and the gravitational forces for such a space.

In [10], the interaction of a field with an electromagnetic potential for an electron is given by

$$
\gamma^{0} \hat{V}=-e \gamma^{\mu} A_{\mu}
$$

where

$$
\hat{V}=-e A^{0} I+e \boldsymbol{\alpha} \cdot \boldsymbol{A}
$$

here $I$ is a $4 \times 4$ unit matrix and $\gamma$ represents the Dirac matrices. The second term on the right of Equation (12) is seen in the Equation (10). The first term on the right of the Equation (12) can be replaced instead of the term $e \Phi$. Then Equation (10) can be written as

$$
\begin{aligned}
i \hbar \frac{\partial \psi}{\partial t}= & {\left[\left[c \boldsymbol{\alpha} \cdot\left(\boldsymbol{p}-\frac{e}{c} \boldsymbol{A}\right)\right]-I \frac{e}{c} A^{0}\right.} \\
& \left.+\beta m c^{2}-2 \frac{Q^{2}}{r} e^{-r / r_{q}}\right] \psi
\end{aligned}
$$

In $[11,12]$, the Lagrangian of the weak local interacttion of the charged leptons is given by

$$
L^{(W)}=2 \sqrt{2} G J_{\lambda}^{(W)}+J^{(W) \lambda}
$$

where $G$ is the universal Fermi constant and the four current is;

$$
\begin{aligned}
J_{v}^{(W)} & =\frac{1}{2}\left[\bar{e} \gamma_{v}\left(1+\gamma^{5}\right) v_{e}+\bar{\mu} \gamma_{v}\left(1+\gamma^{5}\right) v_{\mu}+\bar{\tau}\left(1+\gamma^{5}\right) v_{\tau}\right] \\
& \equiv \frac{1}{2} \sum_{l} \bar{l} \gamma_{v}\left(1+\gamma^{5}\right) v_{l}
\end{aligned}
$$

Here $l \equiv e, \mu, \tau$ for electron, muon, tau respectively and $v_{l} \equiv v_{e}, v_{\mu}, v_{\tau}$ for their neutrinos.

The virial theorem is written in the relativistic form in the articles $[13,14]$. In these articles it was proven that 
the virial theorem has the same validity in the relativistic applications as the applications of it in the classical physics. Therefore, the virial theorem have to be used to convert the Lagrangian of the weak interactions to the form of energy.

In $[15,16]$, the virial theorem states that the potential energy is two times of the kinetic energy in minus sign. Since the Lagrangian in classical mechanics is written by

$$
L=T-V \text {. }
$$

The total energy is $E=T+V$ and by the virial theorem $V=2 T$. Therefore, the total energy is $(3 T)$ and the Lagrangian is $(-T)$. By using these in the Equation (14), the total energy of the weak interactions, in terms of the Lagrangian, can be written as

$$
E=-3\left(2 \sqrt{2} G J_{\lambda}^{(W)}+J^{(W) \lambda}\right)
$$

As a result, the unified equation of interactions is

$$
\begin{aligned}
i \hbar \frac{\partial \psi}{\partial t}= & {\left[c \boldsymbol{\alpha} \cdot\left(\boldsymbol{p}-\frac{e}{c} \boldsymbol{A}\right)-I \frac{e}{c} A^{0}+\beta m c^{2}\right.} \\
& \left.-2 \frac{Q^{2}}{r} e^{-r / r_{q}}-3\left(2 \sqrt{2} G J_{\lambda}^{(W)}+J^{(W) \lambda}\right)\right] \psi
\end{aligned}
$$

The last term in this equation describes the weak interactions. The Equation (18) includes the weak, electromagnetic, gravitational and the strong interactions where the strong and the gravitational interactions are thought to be combined for the considered space.

\section{Results and Discussions}

The wave function of the gravitational and the strong interactions is derived in Equation (7) by putting the Yukawa potential in the Dirac equation, the weak interactions' energy is written by Equation (17) according to the virial theorem, and the unified equation of interactions is derived as Equation (18) to describe the true universe.

\section{Conclusions}

At GUT scale, the high energy structure of the universe needs the validity of the four fundamental forces with the same importance. Since the kinematical and charge effects on the motion could not be eliminated for highly energetic and charged particles at too large or too small distances, the electromagnetic and weak interactions have the same validity at these distances like the gravitational and the strong interactions. In the same way, in the highly energetic space, for large distances the space behaves like a nucleus. Therefore, the strong interactions must be used to define the motion of the particles. For small distances, the highly concentrated mass needs the gravitational forces to explain the attractive forces. Such a true space may be considered as the space the Bing Bang began in. In the world we live in, there is no need to use this equation, as one knows, in special conditions some of these interactions are effective and others are negligible. The application of this equation must be beyond the Standard Model.

As a result, the Dirac equation is a strong candidate for unification of the four fundamental forces, as one is done in this study.

\section{References}

[1] Ali F. Abu Taha, "Universal Gravitation and Formulas of A Unified Interactions," 1993, p. 54. (http://www.shuttlefactor.com/Docs\%20PDF/Universal\% 20Gravitation\%20A.pdf)

[2] J. K. Perring and T. H. R. Skyrme, "A Model Unified Field Equation," Nuclear Physics, Vol. 31, No., 1962, pp. 550-555. doi:10.1016/0029-5582(62)90774-5

[3] Pierre-Henri Chavanis, "Kinetic Equations for Systems With Long-Range Interactions: A Unified Description," 2010, p. 37. arXiv: $1002.3268 v 1$

[4] Tran Hu'u Phat, "Unified Space-Time For Interactions of Elementary Particles," Acta Physica Polonica, Vol. B4, No. 2, 1973, pp. 193-209.

[5] N. Wu, "Unified Theory of Fundamental Interactions," , 2003, p. 23. arXiv.hep-th/0304193v1

[6] M. Ferraris and J. Kijowski, "Unified Geometric Theory of Electromagnetic and Gravitational Interactions," General Relativity and Gravitation, Vol. 14, No. 1, 1982, pp. $37-47$.

[7] J. B. Bjorken and S. D. Drell, "Relativistic Quantum Mechanics," McGraw-Hill, New York, 1964.

[8] J. J. Sakurai, “Advanced Quantum Mechanics," Addison-Wesley, Menlopark, California, 1967.

[9] P. Wagener, "A Unified Theory of Interaction: Gravitation, Electrodynamics and the Strong Force," Pogress in Physics, Vol. 1, No. 33, 2009, pp. 33-35.

[10] W. Greiner, S. Schramm, E. Stein, Quantum Chromodynamics.

[11] A. I. Akhiezer and S. V. Peletminsky, Fields and Fundamental Interactions.

[12] M. Baldo Ceolin, Proceedings of the International School of Physics Enrico Fermi Course LXX.

[13] M. Brack, "Virial Theorems for Relativistic Spin-1/2 and Spin-0 Particles," Physical Review D, Vol. 27, No. 8, 1983, pp. 1950-1953.

[14] F Rosicky and F Mark, "The Relativistic Virial Theorem by the Elimination Method and Nonrelativistic Approximations to This Theorem," Journal of Physics B: Atomic, Molecular and Optical Physics, Vol. 8, No. 16, 1975, pp. 2581-2587.

[15] S. T. Thornton and J. B. Marion, "Classical Dynamics of 
Particles and Systems," In: Chris Hall, Ed., Thomson Learning, Belmont, 2004.

[16] Herbert Goldstein, "Classical Mechanics, Addison-
Wesley Publishing Company, Inc. Reading, Massachusetts, 1959, pp. 69-71. 